\title{
PEDV infection in neonatal piglets through the nasal cavity is mediated by subepithelial $\mathrm{CD}^{+}{ }^{+}$cells
}

\author{
Chen Yuan, Yuxin Jin, Yuchen Li, En Zhang, Penghao Zhang and Qian Yang* (1)
}

\begin{abstract}
Porcine epidemic diarrhea virus (PEDV) primarily infects neonatal piglets causing catastrophic effects on the global pig farming industry. PEDV infects piglets through the nasal cavity, a process in which dendritic cells (DCs) play an important role. However, neonatal piglets have fewer nasal DCs. This study found that subepithelial CD3 ${ }^{+} \mathrm{T}$ cells mediated PEDV invasion through the nasal cavity in neonatal piglets. PEDV could replicate in the nasal epithelial cells (NECs) isolated from the nasal cavity of neonatal piglets. Infection of NECs with PEDV could induce antiviral and inflammatory cytokines at the late stage. The infected NECs mediated transfer of virus to $\mathrm{CD}^{+} \mathrm{T}$ cells distributed in the subepithelial of the nasal cavity via cell-to-cell contact. The infected $\mathrm{CD}^{+} \mathrm{T}$ cells could migrate to the intestine via blood circulation, causing intestinal infection in neonatal piglets. Thus, the findings of this study indicate the importance of $C D 3^{+} T$ cells in the dissemination of PEDV from the nasal cavity to the intestinal mucosa in neonatal piglets.
\end{abstract}

Keywords: PEDV, CD3 ${ }^{+}$T cells, nasal cavity, neonatal piglets

\section{Introduction}

The respiratory tract is the primary pathway for various respiratory pathogens, such as those causing influenza and pneumonia $[1,2]$. As a gateway to the respiratory tract, the nasal cavity plays a vital role as the first line of defense against the invasion of such microorganisms [3]. In addition, increasing evidence for the air transmission of gastrointestinal pathogens, including Norwalk viruses and rotavirus, has been reported $[4,5]$. The interactions between respiratory viruses and host nasal epithelial cells (NECs) pattern recognition receptors, may induce the release of cytokines/chemokines and stimulate an antiviral response [6]. However, little is known about the characteristics of gastrointestinal viral infection in NECs.

${ }^{*}$ Correspondence: zxbyq@njau.edu.cn

MOE Joint International Research Laboratory of Animal Health and Food Safety, College of Veterinary Medicine, Nanjing Agricultural University,

Weigang 1, Nanjing, Jiangsu 210095, China
Porcine epidemic diarrhea virus (PEDV) is the causative agent of porcine epidemic diarrhea (PED), an acute and highly contagious enteric viral disease $[7,8]$. Although PEDV can occur in swine of any age, neonatal piglets are the most severely affected $[9,10]$. Symptoms in infected piglets include watery diarrhea, dehydration, and vomiting. The fecal-oral route is believed to be the primary mode of PEDV transmission [11]. Recent studies established an alternative pathway of enteric PEDV dissemination from the nasal cavity to the intestinal mucosa in swine [12]. However, the interactions between enteric PEDV and host NECs have not been elucidated. In addition, dendritic cells (DCs) located beneath the nasal mucosa of piglets can capture PEDV by forming transepithelial dendrites during PEDV intranasal infection [12]. Thus, DCs harboring PEDV may be vehicles for the dissemination of the virus in PEDV infection. However, neonatal piglets have fewer nasal DCs compared to swine of any older age [13]. Thus, other immune cells may 
participate in PEDV infection in neonatal piglets through nasal spray.

The nasal cavity of piglets is categorized into three parts: the regio vestibularis (I, II), regio respiratoria (III, IV), and regio olfactoria (V). Lymphoid tissue is randomly located in the nasal cavity. Lymphocytes have been detected in the subepithelium of the nasal cavity [14]. Moreover, it has been reported that $\mathrm{T}$ lymphocytes could carry PEDV [12]. The motility of lymphocytes can be altered during viral infection [15]. PEDV causes severe enteric disease in neonatal piglets, as well as milder

Table 1 Primers used for real-time PCR

\begin{tabular}{|c|c|c|}
\hline Gene & Primer sequence $\left(5^{\prime}-3^{\prime}\right)$ & Orientation \\
\hline \multirow[t]{2}{*}{ IFN-a } & СТCTTCCTCCAGAAACCTGCAA & Forward \\
\hline & GAGGAAGAATGGGCTTGTTAGTC & Reverse \\
\hline \multirow[t]{2}{*}{ IFN- $\beta$} & CCACCACAGCTCTTTCCATGA & Forward \\
\hline & TGAGGAGTCCCAGGCAACT & Reverse \\
\hline \multirow[t]{2}{*}{$\mid L-1 \beta$} & AGAGGGACATGGAGAAGCGA & Forward \\
\hline & GCCCTCTGGGTATGGCTTT & Reverse \\
\hline \multirow[t]{2}{*}{ TNF-a } & GCCCTTCCACCAACGTTTTC & Forward \\
\hline & TCCCAGGTAGATGGGTTCGT & Reverse \\
\hline \multirow[t]{2}{*}{ |L-6 } & CCTCGGCAAAATCTCTGCAA & Forward \\
\hline & TGAAACTCCACAAGACCGGT & Reverse \\
\hline \multirow[t]{2}{*}{ IL-8 } & СCTCATTCCTGTGCTGGTCA & Forward \\
\hline & TGCAAGTTGAGGCAAGAAGAC & Reverse \\
\hline \multirow[t]{2}{*}{ |L-10 } & CGGCCCAGTGAAGAGTTTCT & Forward \\
\hline & TGCCTTCGGCATTACGTCTT & Reverse \\
\hline \multirow[t]{2}{*}{ TLRI } & AGATTTCGTGCCACCCTATG & Forward \\
\hline & CCTGGGGGATAAACAATGTG & Reverse \\
\hline \multirow[t]{2}{*}{ TLR2 } & GAGTCTGCCACAACTCAAAGA & Forward \\
\hline & CAGAACTGACAACATGGGTAGAA & Reverse \\
\hline \multirow[t]{2}{*}{ TLR3 } & GAGCAGGAGTTTGCCTTGTC & Forward \\
\hline & GGAGGTCATCGGGTATTTGA & Reverse \\
\hline \multirow[t]{2}{*}{ TLR4 } & TCATCCAGGAAGGTTTCCAC & Forward \\
\hline & TGTCCTCCCACTCCAGGTAG & Reverse \\
\hline \multirow[t]{2}{*}{ TLR5 } & GGTCCCTGCCTCAGTATCAA & Forward \\
\hline & TGTTGAGAAACCAGCTGACG & Reverse \\
\hline \multirow[t]{2}{*}{ TLR6 } & TCAAGCATTTGGACCTCTCA & Forward \\
\hline & TTCCAAATCCAGAAGGATGC & Reverse \\
\hline \multirow[t]{2}{*}{ TLR7 } & TCTGCCCTGTGATGTCAGTC & Forward \\
\hline & GCTGGTTTCCATCCAGGTAA & Reverse \\
\hline \multirow[t]{2}{*}{ TLR8 } & CTGGGATGCTTGGTTCATCT & Forward \\
\hline & CATGAGGTTGTCGATGATGG & Reverse \\
\hline \multirow[t]{2}{*}{ TLR9 } & AGGGAGACCTCTATCTCCGC & Forward \\
\hline & AAGTCCAGGGTTTCCAGCTT & Reverse \\
\hline \multirow[t]{2}{*}{ TLRIO } & GCCCAAGGATAGGCGTAAAT & Forward \\
\hline & CTCGAGACCCTTCATTCAGC & Reverse \\
\hline \multirow[t]{2}{*}{$\beta$-actin } & TGCTGTCCCTGTATGCCTCT & Forward \\
\hline & CTTTGATGTCACGCACGATTT & Reverse \\
\hline
\end{tabular}

disease in older weaned pigs. In early life, innate immune responses are not sufficiently competent to clear most pathogens or to prevent the dissemination of infections [16]. Therefore, we hypothesized that $\mathrm{T}$ lymphocytes may participate in PEDV infection after PEDV intranasal inoculation in neonatal piglets.

The results of the present study support an alternative pathogenic pathway of PEDV, which results in typical diarrhea symptoms in neonatal pigs. In this pathway, $\mathrm{CD}^{+} \mathrm{T}$ cells mediate PEDV infection through the nasal cavity in neonatal piglets. Our results revealed the mechanism of intranasal inoculation of PEDV in neonatal piglets, which can describe the development of strategies that are effective in controlling PEDV epidemics.

\section{Materials and methods Reagents and cell lines}

Anti-pig PE-SWC3a was purchased from Abcam. Antipig FITC-MHCII was purchased from Bio-Rad. Dylight 488-, 594-, -conjugated secondary antibodies were purchased from MultiSciences (Lianke) Biotech Co., Ltd. Anti-pig APC-CD3 $\varepsilon$ was purchased from BD Biosciences. Human placenta Type IV was purchased from Sigma. The anti-PEDV $\mathrm{N}$ protein $\mathrm{mAb}$ was purchased from Medgene labs. Anti-pig epithelial cell marker PE- Keratin 18 (CK18) mAb was purchased from Novus Biologicals. Anti-APC (130-097-143) MiniMACS Starting kits were purchased from Miltenyi Biotec. Vero E6 cells (ATCC CCL81) were kindly provided by the Veterinary Medicine Research Center of the Da Bei Nong Group. The cell line was regularly tested for mycoplasma contamination.

\section{Animals}

Conventional Duroc $\times($ Landrace $\times$ Yorkshire $)$ neonatal piglets (1-day-old) were obtained from a swine herd at the Jiangsu Academy of Agricultural Science. The neonatal piglets were born via natural farrow and fed synthetic milk. The swine herd was seronegative for antibodies against PEDV, porcine reproductive and respiratory syndrome (PRRSV), transmissible gastroenteritis virus (TGEV) and porcine circovirus type 2 (PCV2). Each experimental group of neonatal piglets was housed in a separate room with constant humidity and temperature and a $12 \mathrm{~h}$ light/ dark cycle. All procedures and experiments performed on animals were approved by the Institutional Animal Care and Use Committee of Nanjing Agricultural University and followed the National Institutes of Health guidelines for animal experiments' performance.

\section{Virus}

The wild-type PEDV strain Zhejiang08 was preserved in our laboratory. The virus clustered with the emerging virulent strain [12]. 


\section{Isolation of NECs and in vitro infection with PEDV}

Isolated NECs were cultured as previously described [12]. To isolate NECs from the nasal mucosa of neonatal piglets (1-day-old), the mucosa was cut into $1-2 \mathrm{~cm}$ pieces. The pieces were washed with Hank's balanced salt solution (HBSS) at least five times and digested in a solution containing minimal essential medium supplemented with $50 \mu \mathrm{g} / \mathrm{mL}$ gentamicin, $1.25 \mu \mathrm{g} / \mathrm{mL}$ amphotericin, $1.4 \mathrm{mg} /$ $\mathrm{mL}$ pronase, $100 \mu \mathrm{g} / \mathrm{mL}$ DNase and $1 \%$ penicillin-streptomycin at $4{ }^{\circ} \mathrm{C}$ for $21 \mathrm{~h}$. Cell were obtained by centrifugation. Cell suspensions were transferred to a T25 tissue culture flask and incubated in DMEM containing $4 \%$ fetal bovine serum at $37^{\circ} \mathrm{C}$ for $3 \mathrm{~h}$ to allow attachment of fibroblasts. The cell suspension was centrifuged at $120 \times g$ for $6 \mathrm{~min}$ and the resultant pellet was resuspended in complete bronchial epithelial growth media. The acquired NECs were seeded $\left(1 \times 10^{5}\right)$ in wells of Transwell devices coated with human placenta Type IV collagen $(6 \mu \mathrm{g} / \mathrm{mL})$. The medium was replaced every second day. When $80 \%$ confluency was reached, the NECs were washed in PBS and then infected with PEDV at a multiplicity of infection (MOI) of 0.1. The purity of the NECs was determined by fluorescence-activated cell sorting (FACS). At $1 \mathrm{~h}$ postinfection (hpi), virus-containing medium was removed, the cells were washed twice with PBS, and fresh medium was added to each well. The NECs were collected at different timepoints for subsequent cytokine/Toll-like receptor (TLR) and viral analyses.

\section{Generation of $\mathrm{CD}^{+} \mathrm{T}$ cells}

The nasal mucosa was cut into $0.5 \mathrm{~cm}$ pieces. The pieces were incubated in $20 \mathrm{~mL}$ of $10 \mathrm{mM}$ EDTA in HBSS for $20 \mathrm{~min}$ at $4{ }^{\circ} \mathrm{C}$. The samples were then centrifuged, discarded, and placed in digestion solution containing $4 \%$ fetal bovine serum, $2 \mathrm{mg} / \mathrm{mL}$ each of collagenase $\mathrm{D}$ and DNase I, and $100 \mathrm{U} / \mathrm{mL}$ dispase and slowly rotated at $37^{\circ} \mathrm{C}$ for $20 \mathrm{~min} . \mathrm{CD}^{+}$cells were obtained by density gradient centrifugation, sorted by anti-allophycocyanin (APC) microbeads, and activated by phytohemagglutinin and interleukin-2 (IL-2) for $3 \mathrm{~d}$ for subsequent experiments.

\section{PEDV infection and transmission}

NECs were infected with PEDV $(\mathrm{MOI}=0.1)$ at $37^{\circ} \mathrm{C}$. At $1 \mathrm{hpi}$, the cells were washed extensively to remove the unbound virus. The PEDV infected NECs were cocultured with $\mathrm{CD}^{+} \mathrm{T}$ cells isolated from nasal mucosa at $37^{\circ} \mathrm{C}$. At $4 \mathrm{hpi}$, the number of $\mathrm{CD}^{+} \mathrm{T}$ cells harboring PEDV was determined by flow cytometry.

\section{PEDV intranasal inoculation}

Neonatal piglets (1-day-old) of similar weight were randomly allocated into two groups (negative control [I] and
PEDV infected [II] groups; $\mathrm{n}=6$ per group). The groups were housed in two separate rooms in a high-security isolation facility. Neonatal piglets in group II were challenged with $1 \mathrm{~mL}$ PEDV $\left(10^{6}\right.$ plaque forming units per $\left.\mathrm{mL}\right)$ by nasal inoculation. The nasal spray device used for nasal inoculation is commonly used for vaccine absorption by the nasal mucosa and effectively atomizes particles. In group I, the same volume of PBS was inoculated in the same manner. The animals were fed with synthetic milk every $3 \mathrm{~h}$ throughout the experiment to meet or exceed the National Research Council requirements for nutrients and energy for this size of piglets. After challenge, neonatal piglets were observed daily for symptoms of diarrhea. Nasal cavity tissue of neonatal piglets ( $\mathrm{n}=3$ for each group) was sampled and processed for immunohistochemistry (IHC) analysis $12 \mathrm{~h}$ after the nasal challenge. At the conclusion of the experiment, the piglets were euthanized by intravenous injection of pentobarbital sodium $(100 \mathrm{mg} / \mathrm{kg})$. Jejunum tissues were collected for IHC and immunofluorescence (IF) viral analyses.

\section{Quantitative reverse transcription-PCR (RT-qPCR)}

Total RNA from NECs was purified using RNAiso Plus kit (TaKaRa Bio, Dalian, China) following the manufacturer's instructions. Fresh RNA $(1 \mu \mathrm{g})$ was used as a template to synthesize first-strand cDNA with commercial oligo dT primers using Prime Script ${ }^{\mathrm{TM}}$ II 1st strand cDNA Synthesis Kit (TaKaRa Bio). PCR was performed using a SYBR Green qPCR Kit (TaKaRa Bio) in an Applied Biosystems 7500 Fast Real-Time PCR System (Life Technologies). Specific primers are shown in Table 1. Gene expression was normalized to amplify glyceraldehyde 3-phosphate dehydrogenase (GAPDH). The data were analyzed using the $2^{-\Delta \Delta \mathrm{CT}}$ method.

\section{Western blot analysis}

Total protein from different tissues or NECs was obtained following lysis using lysis buffer. Proteins in the lysates were separated by $10 \%$ sodium dodecyl sulfate-polyacrylamide gel electrophoresis (SDS-PAGE) and transferred to a polyvinylidene difluoride (PVDF) membrane (Bio-Rad, CA). After blocking with 5\% nonfat milk in Tris-buffered saline containing 0.05\% Tween-20 (TBST), the membrane was incubated with the particular primary antibody, followed by horseradish peroxidase-conjugated secondary antibodies in blocking reagent. After extensive washing with TBST, immune reactive bands were analyzed by film exposure after enhanced chemiluminescence reaction (Millipore, Bedford, MA, USA).

\section{Flow cytometry}

$\mathrm{CD}^{+} \mathrm{T}$ cells were acquired from the blood of neonatal piglets or after co-cultivation with NECs. The surface of each cell was stained with the indicated antibody. The 
cells were resuspended in fixation/permeabilization solution (BD Cytofix/Cytoperm kit; BD Pharmingen) and stained with PEDV N protein antibody to detect intracellular PEDV. After three washes with PBS, the cells were phenotypically analyzed by FACS.

\section{IHC and IFA assays}

After fixation, histological sections of five blocks of nasal cavity tissue were selected according to the fractions $1 / 20,1 / 4,2 / 5,3 / 5$, and $4 / 5$. Five cross-sections (I, II, III, $\mathrm{IV}$, and $\mathrm{V}$ ) of each fraction were subsequently examined by IHC to assess the distribution of PEDV using primary antibody directed against PEDV N protein. For IF staining of PEDV, tissue sections were permeabilized in $0.4 \%$ Triton X-100 in PBS for 5 min. After treatment with 5\% bovine serum albumin in PBS for $1 \mathrm{~h}$, PEDV located in the nasal or jejunal mucosa was immunolabeled with PEDV $\mathrm{N}$ protein overnight at $4{ }^{\circ} \mathrm{C}$, followed by Alexa Fluor 488-conjugated goat anti-mouse antibody. Colonization of PEDV in the jejunum was detected by IFA with PEDV polyclonal antibody.

\section{Statistical analyses}

Results are expressed as mean $\pm \mathrm{SD}$ and analyzed using SPSS 17.0. One-way analysis of variance (ANOVA) was employed to determine significant differences among multiple groups. The t-test was employed to determine the differences between the two groups. Significance was expressed at a $P$-value $<0.05$ or $<0.01$. Data were combined from at least three independent experiments, unless otherwise stated.

\section{Results}

\section{Replication of PEDV in NECs}

NECs were successfully isolated from the nasal cavity of neonatal piglets as demonstrated by morphological examination of cultured cells. The cilia structure in some of the isolated nasal epithelium was preserved (Additional file 1A). The cytokeratin 18 epithelial cell marker was used to identify the purity of the isolated NECs. FACS analysis indicated that at least $94.6 \%$ of the cell population comprised cytokeratin-positive epithelial cells (Additional file 1B). To detect whether PEDV replicated in NECs, the cells were inoculated with PEDV $(\mathrm{MOI}=0.1)$. At 6, 12, 24, 36, 48, and $60 \mathrm{hpi}$, NECs and supernatants were collected for RT-qPCR and western blot analyses, and determination of median tissue culture infectious dose $\left(\mathrm{TCID}_{50}\right)$ to determine intracellular and extracellular virus titers. RT-qPCR showed that PEDV gene copies reached a peak of $4.12 \log _{10}$ within 12 hpi and declined at $24 \mathrm{hpi}$ (Figure $1 \mathrm{~A}$ ). TCID $_{50}$ results revealed that viral gene replication occurred prior to virus release. The virus titer peaked at $24 \mathrm{hpi}$ and then decreased gradually (Figure 1B). Western blot analysis results were similar to the $\mathrm{TCID}_{50}$ results. PEDV N protein was increased at 12 and $24 \mathrm{hpi}$, and then decreased gradually (Figure $1 \mathrm{C}$ ). The collective findings indicated the replication of PEDV in NECs.

\section{PEDV infection induces antiviral and cytokines expression in NECs}

Cytokine mRNA expression levels were detected by RTqPCR and normalized to the expression of the GAPDH gene at different timepoints. As shown in Figure 2, compared with the control group, the PEDV infected group had significantly increased mRNA expression levels of interferon (IFN)- $\alpha$, IFN- $\beta$, (IL)-1 $\beta$, IL-6, IL-8, IL-10, and tumor necrosis factor (TNF)- $\alpha$. The mRNA expression levels of TNF- $\alpha$, IL-6, IL-8, and IL-10 were significantly upregulated at $36 \mathrm{hpi}$. The mRNA expression levels of IFN- $\alpha$, IFN- $\beta$, and IL- $1 \beta$ were significantly upregulated at 48 hpi.

\section{Influence of PEDV infection on expression of TLR1-10 genes in NECs}

The mRNA expression levels of the genes encoding TLR1-10 in PEDV infected NECs were determined by RT-PCR at various timepoints following infection. As shown in Figure 3, the expression of the TLR1, TLR2, TLR3, TLR4, TLR6, TLR7, and TLR8 genes at 24, 36, 48 , and 60 hpi were significantly higher than those in the control group, and their expression levels were significantly higher at $48 \mathrm{hpi}$ than those at 24,36 , and $60 \mathrm{hpi}$, except for TLR2. The expression of the TLR5 gene at 6 , 12 , 24, and 36 hpi was significantly lower than those in the control group. Expression levels of the TLR9 and TLR10 genes were significantly higher at 24 hpi compared to those in the control group.

\section{Distribution of PEDV in nasal cavity after intranasal inoculation in neonatal piglets}

To detect the distribution of PEDV in the nasal cavity of neonatal piglets, the NECs were isolated via staining with antibody specific cytokeratin 18 after intranasal inoculation for $12 \mathrm{~h}$ and subjected to FACS. FACS analyses revealed that $4.86 \%$ of the NECs were positive for cytokeratin 18 (Figure 4A). IHC of nasal cavity tissue acquired at $12 \mathrm{hpi}$ revealed an uneven distribution of virus-positive cells in regions I-IV of the nasal cavity, with the most virus-positive cells observed in region IV (Figures 4B, C).

\section{PEDV infection of $\mathrm{CD}^{+} \mathrm{T}$ cells distributed} in the subepithelium of the nasal cavity in neonatal piglets Submucosal DCs in the nasal cavity can capture PEDV and transfer the virus to $T$ cells in piglets [12]. However, 
A

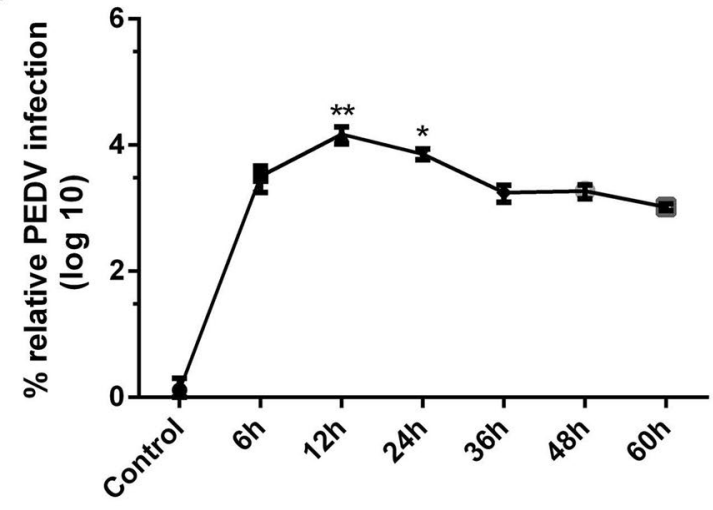

B

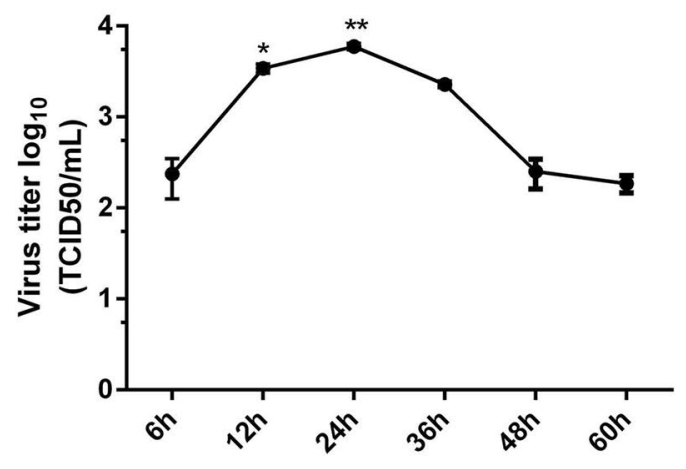

C

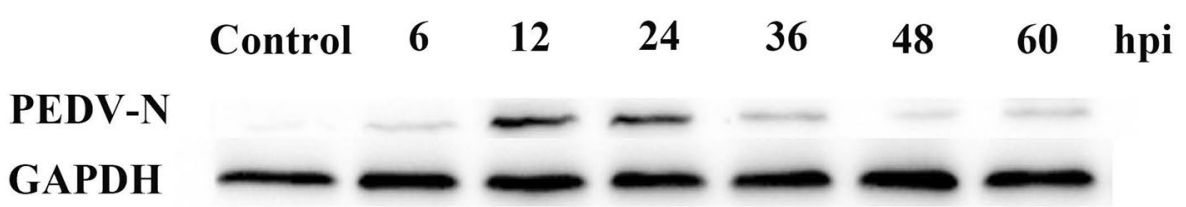

Figure 1 Replication of PEDV in NECs. A RNA expression levels of PEDV infected NECs at different time points was evaluated by RT-qPCR. B Culture supernatants of PEDV infected NECs were collected at different time points. The viral titer in the supernatant was determined with Vero cells with the results expressed as $T C I D_{50} / \mathrm{mL}$. C Protein expression of PEDV in the NECs at different time points by western blotting with a mouse $\mathrm{mAb}$ against $\mathrm{N}$ protein. ${ }^{*}$ Represents a significant difference relative to the $6 \mathrm{~h}$ group $(P<0.05)$, and ${ }^{* *}$ Represents an extremely significant difference relative to the 6 h group $(P<0.01)$.

submucosal DCs and in the soft palate tonsil, nasopharyngeal tonsil and lingual tonsil are sparse in neonatal piglets [13]. We examined by IF the distribution patterns of submucosal DCs in regions IV of the nasal cavity from neonatal piglets using dual staining with antibodies specific to DCs markers. The double-positive Swc3a ${ }^{+} \mathrm{MHC} \mathrm{II}^{+} \mathrm{DCs}$ stained yellow or orange. There were few DCs in the nasal cavity (Figure 5A). The finding indicates that PEDV could infect neonatal piglets via other immune cells by intranasal inoculation. IHC was used to examine the distribution patterns of $\mathrm{CD}^{+} \mathrm{T}$ cells in the nasal cavity. $\mathrm{CD}^{+} \mathrm{T}$ cells were distributed in the subepithelium of the nasal cavity from neonatal piglets, with increasing numbers of cells from the proximal to distal side of the nasal cavity (Figure 5B, C). $\mathrm{CD}^{+} \mathrm{T}$ cells derived from the nasal cavity of neonatal piglets through intranasal inoculation could contain PEDV, as visualized by double IF staining of $\mathrm{CD}^{+} \mathrm{T}$ cell and PEDV proteins (Figure 5D). These results demonstrated that $\mathrm{CD}^{+} \mathrm{T}$ cells present in the subepithelium of the nasal cavity from neonatal piglets were infected by PEDV.

\section{$\mathrm{CD}^{+} \mathrm{T}$ cells acquire PEDV from NECs and migrate} to the intestine of neonatal piglets via blood circulation To detect whether PEDV-harboring NECs allow the virus to be transferred to $\mathrm{CD}^{+}{ }^{+} \mathrm{T}$ cells beneath the NECs, a co-culture system between the nasal epithelium and $\mathrm{CD}^{+} \mathrm{T}$ cells was established in vitro (Figure 6A). NECs infected with PEDV were co-incubated with $\mathrm{CD}^{+}{ }^{+} \mathrm{T}$ cells sorted by anti-APC microbeads. PEDV was detected in $\mathrm{CD}^{+} \mathrm{T}$ cells after $4 \mathrm{~h}$ of co-culture (Figure 6B). FACS analysis revealed that the percentage of PEDV-positive $\mathrm{CD}^{+}{ }^{+} \mathrm{T}$ cells was $1.79 \%$ and $1.68 \%$ at $12 \mathrm{hpi}$ in peripheral blood mononuclear cells (PBMC) and jejunum of neonatal piglets after PEDV intranasal inoculation, respectively (Figure 6C). To confirm the FACS analysis results, we used confocal microscopy to visualize the subcellular locations of PEDV and $\mathrm{CD}^{+} \mathrm{T}$ cells in the intestine of neonatal piglets after intranasal infection at $12 \mathrm{hpi}$. IFA revealed the presence of $\mathrm{CD}^{+} \mathrm{T}$ cells carrying PEDV (Figure 6D). These results showed that PEDV-carrying NECs allow the virus to be transferred to $\mathrm{CD}^{+} \mathrm{T}$ cells beneath the NECs in neonatal piglets. Subsequently, PEDV-carrying $\mathrm{CD}^{+} \mathrm{T}$ cells migrate to the intestine of neonatal piglets via blood circulation.

\section{PEDV intranasal inoculation in neonatal piglets causes typical PED symptoms}

To verify that PEDV could infect neonatal piglets through intranasal inoculation, we carried out challenge experiments. The neonatal piglets were randomly divided into the control and PEDV intranasal 


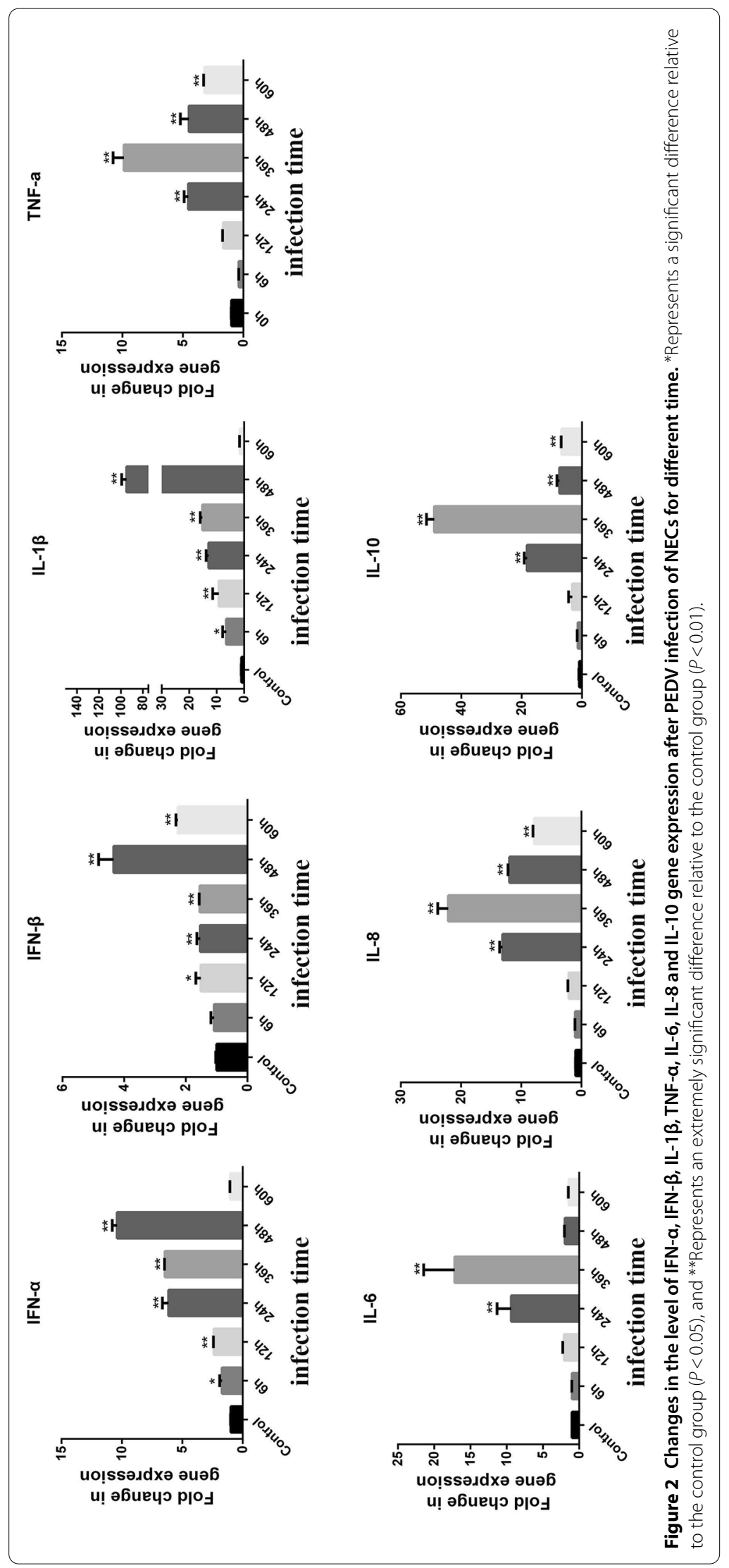




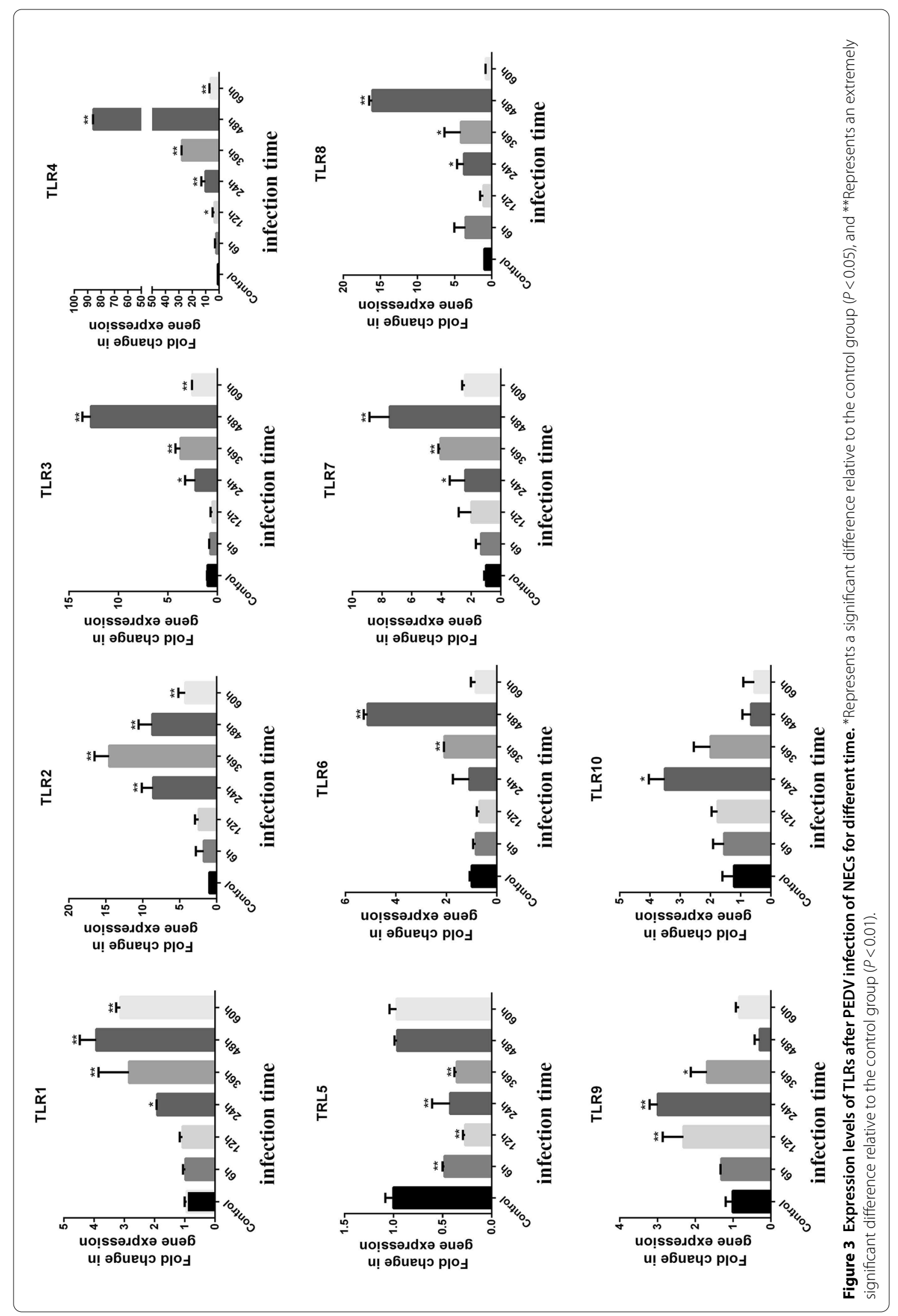


A
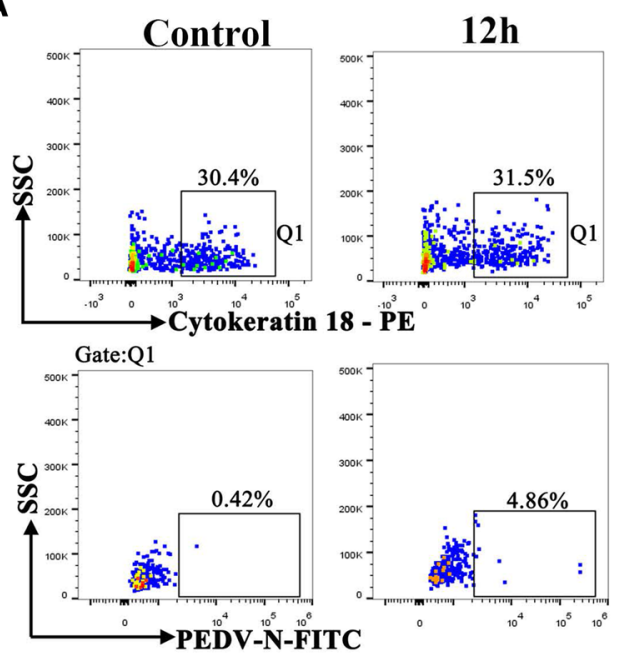

C

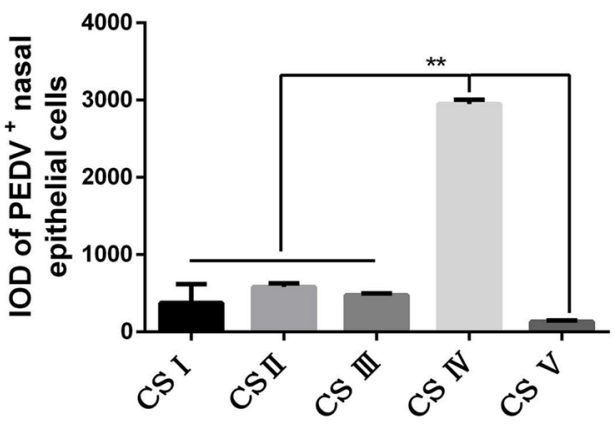

B

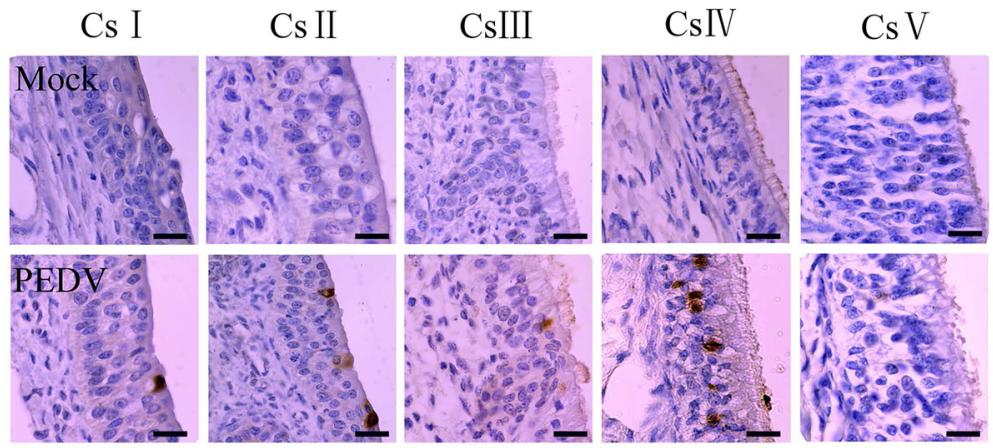

Figure 4 The distribution of PEDV in nasal cavity after intranasal inoculation in neonatal piglets. A For FACS analyses, neonatal piglets were nasally administered PEDV at indicated times. Then, individual cells isolated from the nasal mucosa were gated based on cytokeratin 18, and viral infection was detected by PEDV N protein staining, $\mathrm{n}=3$ from 3 piglets per group. $\mathbf{B} \| \mathrm{HC}$ results showed PEDV distribution in five cross-sections (I, $\mathrm{II}, \mathrm{II}, \mathrm{IV}$ and V) of the nasal cavity at $12 \mathrm{~h}$ post intranasal infection. The scale bar represents $20 \mu \mathrm{m}$. C Quantitative analysis of PEDV positive cells in the nasal cavity. All data shown are the mean results \pm SD from three independent experiments. Statistical significance was tested using one-way ANOVA. ${ }^{* *} P<0.01$.

inoculation groups ( $\mathrm{n}=3$ per group). Severe watery diarrhea and vomiting were first detected in the PEDV inoculated neonatal piglets at $56 \mathrm{hpi}$. At $60 \mathrm{hpi}$, these piglets began to exhibit classical PEDV symptoms, including acute, severe watery diarrhea, depression, and lethargy. Abundant yellow, foul smelling watery stools were also observed around the perianal region of neonatal piglets (Figure 7A). The neonatal piglets were then euthanized. Pathological changes noted on autopsy of the group II animals included thinning and neartransparency of the walls of the small intestines and extended stomach filled with curdled milk (Figure 7B). Histopathological examination showed severe diffuse atrophy, fusion of villi of the small intestine, hemorrhage, and a number of inflammatory cells in group II
(Additional file 2). RT-qPCR showed viral RNA expression in different tissues of neonatal piglets after PEDV intranasal inoculation. PEDV mainly colonized the jejunum and peak viral RNA titers reached $4.79 \mathrm{log}$. The viral RNA titers in the jejunum were significantly higher than those in the other tissues (Figure 7C). Western blot results further validated the PEDV level in different tissues, and a significant quantity of PEDV $\mathrm{N}$ protein was detected in the jejunum and ileum. However, no immune reactivity was observed with proteins of the trachea or stomach (Figure 7D). IHC and IF analyses revealed many PEDV-positive cells in the jejunum (Figures 7E, F). These results indicate that intranasal inoculation of PEDV in neonatal piglets causes PEDV intestinal infection. 
A

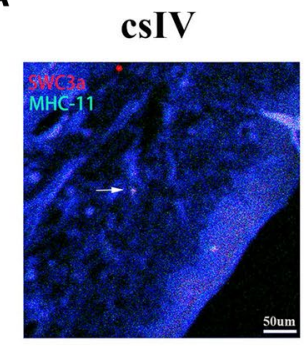

C

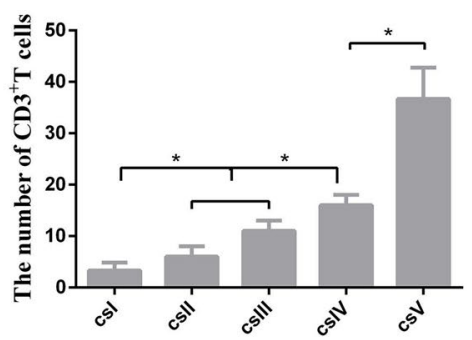

B

esI

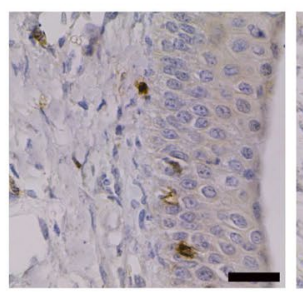

D

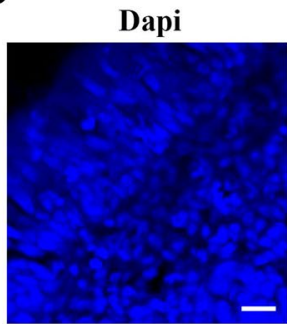

csII

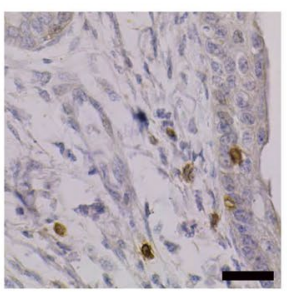

CD3

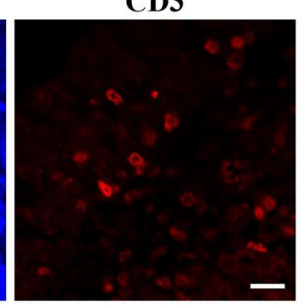

csIII

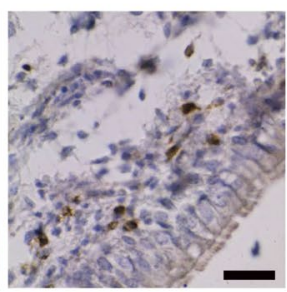

PEDV

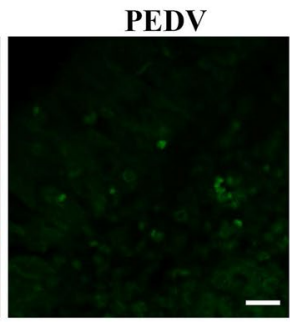

esIV

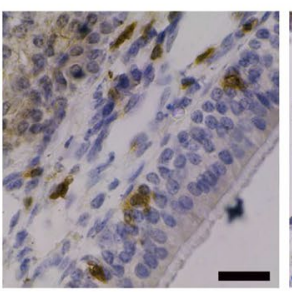

Merge

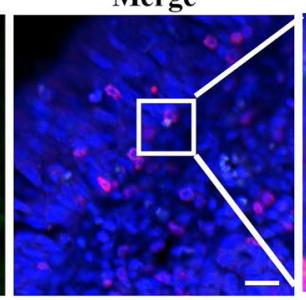

csV
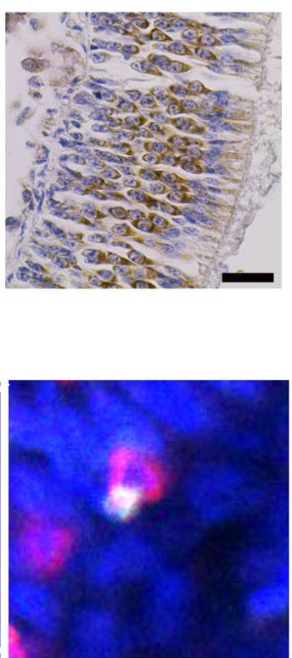

Figure 5 The distribution pattern of $\mathrm{DCs}$ and $\mathrm{CD}^{+} \mathrm{T}$ cells in the nasal cavity of neonatal piglets. $\mathrm{A} I \mathrm{~F}$ analysis of $\mathrm{MHCI}{ }^{+} \mathrm{SWC} \mathrm{a}^{+} \mathrm{DCs}$ in the nasal cavity from neonatal piglets. The scale bar represents $50 \mu \mathrm{m}$. B CD3 ${ }^{+} \mathrm{T}$ cells were detected in the nasal cavity from neonatal piglets using $\mathrm{IHC}$. The positive cells were stained brown. The scale bar represents $20 \mu \mathrm{m}$. C Quantitative analysis of $\mathrm{CD} 3^{+} \mathrm{T}$ cells in the nasal cavity. The number of $\mathrm{CD}^{+} \mathrm{T}$ cells was counted from five cross-sections in a unit area $(40 \mathrm{x}){ }^{*} P<0.05$. D IF analysis examined PEDV in $\mathrm{CD}^{+} \mathrm{T}$ cells of the nasal cavity from neonatal piglets through intranasal inoculation. The scale bar represents $20 \mu \mathrm{m}$. Blue, DAPI; green, PEDV; Red, CD3.

\section{Discussion}

The nasal cavity is one of the main routes of infection by pathogenic microorganisms in animals and humans [17]. Various respiratory viruses invade the nasal cavity and spread within the body. Once pathogens reach the NECs, these cells rapidly recognize the invaders and initiate local and systemic immune responses [18].The interactions between the respiratory viruses and host NECs have been reported. For example, influenza virus A infection in NECs can stimulate the expression of chemokines eotaxin [19]. NECs and bronchial epithelial cells have similar antiviral and pro-inflammatory responses during rhinovirus infection [20]. Cytokines play a vital role in protecting hosts from viral infection. However, little is known about the characteristics of gastrointestinal viral infection in NECs. The expression of the pro-inflammatory cytokines IL- $\alpha$, IL-1 $\beta$, and TNF- $\alpha$ are significantly upregulated in Vero cells infected with PEDV [21]. PEDV infection activates nuclear factor-kappa B (NF-kB) through the TLR2, TLR3, and TLR9 pathways in porcine intestinal epithelial cells [22]. In addition, PEDV infection can induce innate immune responses in intestinal porcine jejunum epithelial cells, leading to changes in the expression of TLRs and the release of downstream cytokines [23].

In this study, the response of NECs nasal during PEDV infection was studied. The expression levels of IFN- $\alpha$, IFN- $\beta$, and IL- $1 \beta$ in the cells at different infection times were higher than those in the control group, especially at $48 \mathrm{hpi}$. The expression levels of TNF- $\alpha$, IL-6, IL-8, and IL-10 at different infection time points were higher than those in the control group, especially at 36 hpi. The effects of PEDV infection on the expression of TLR genes were also analyzed. The expression levels of most TLR family genes were significantly higher than those in the control group at different times. Contrarily, expression of TLR5 decreased with time. These results suggest the importance of cytokines 
A

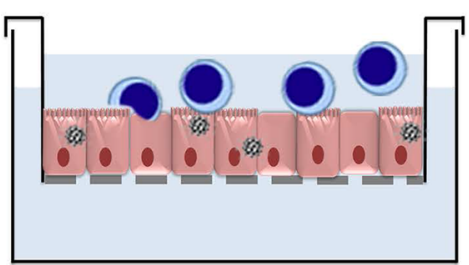

CD3 ${ }^{+} \mathrm{T}$ cells

nasal epithelical cells

3EDV

B
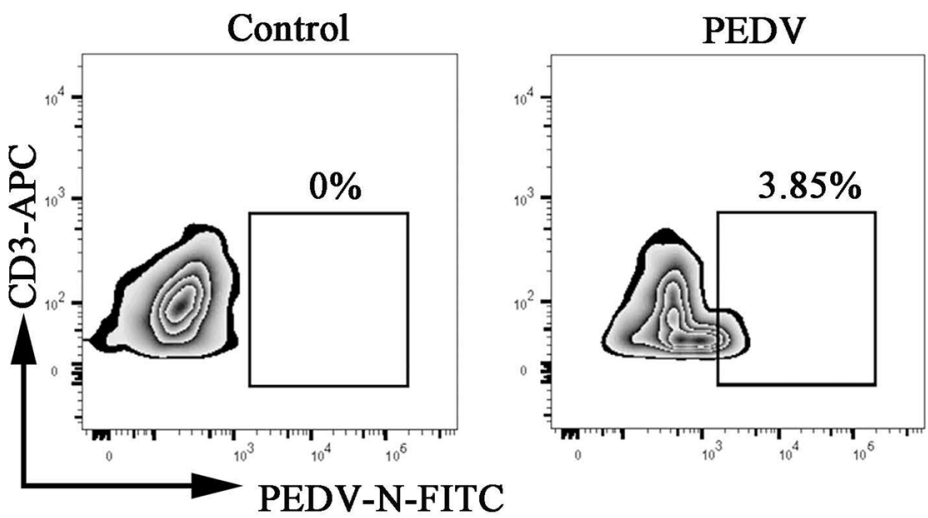

C
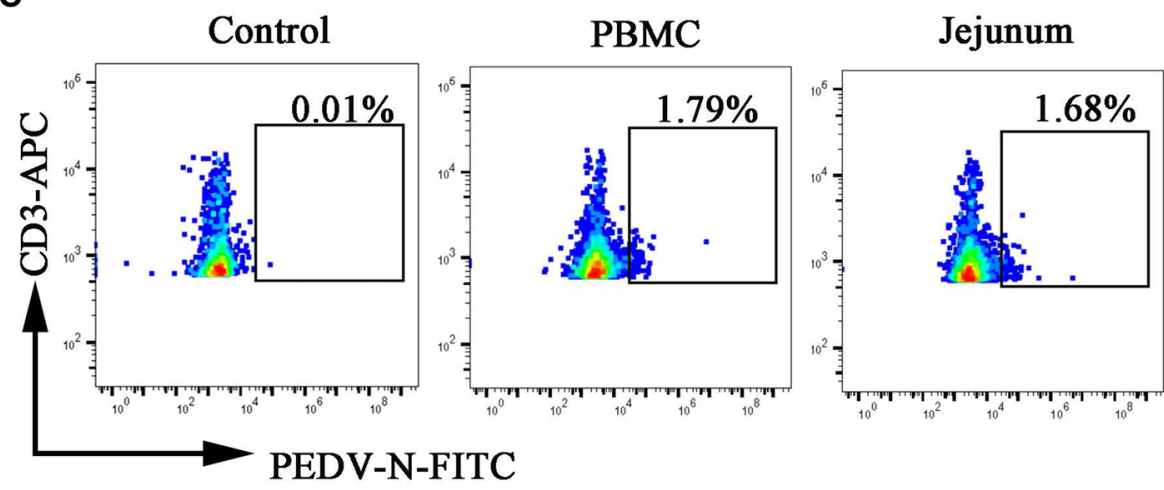

D

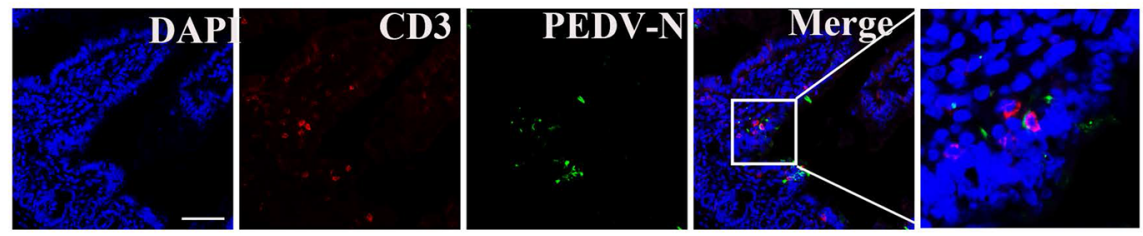

Figure6 $\mathrm{CD}^{+} \mathrm{T}$ cells acquired PEDV from NECs and migrated to the intestine of neonatal piglets via blood circulation. A Schematic model of PEDV-carrying NECs transmission of the virus to T cells. T cells were co-cultured with NECs by contact. B PEDV-infected NECs were co-cultured with $\mathrm{CD}^{+} \mathrm{T}$ cells for $4 \mathrm{~h}$. Viral infection in $\mathrm{CD}^{+} \mathrm{T}$ cells was detected by PEDV N protein staining. $\mathrm{C}$ FACS analyzed the number of PEDV-loaded $\mathrm{CD}^{+} \mathrm{T}$ cells derived from PBMC and the jejunum in neonatal piglets at $12 \mathrm{hpi}$ after intranasal inoculation. $\mathbf{D}$ IF analysis examined CD3 ${ }^{+} \mathrm{T}$ cells carrying PEDV of jejunum in neonatal piglets through intranasal inoculation. The scale bar represents $50 \mu \mathrm{m}$. Blue, DAPI; green, PEDV; Red, CD3.

and TLR gene expression in PEDV infection. These results agree with previous reports on PEDV infected epithelial cells [23]. The changes in TLR genes and the expression of cytokines in NECs infected with PEDV were preliminarily investigated to provide a theoretical reference and experimental basis for further study on the role of TLR genes in PEDV infection.

Multiple types of immune cells exist beneath the mucosal epithelium of the nasal cavity. These cells effectively prevent invasion and infection by pathogenic microorganisms [24, 25]. Paradoxically, submucosal 
A

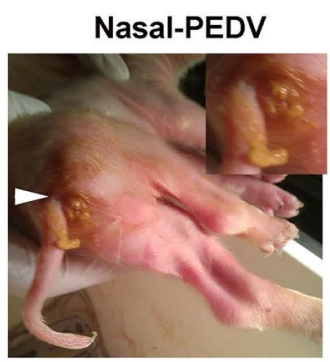

C

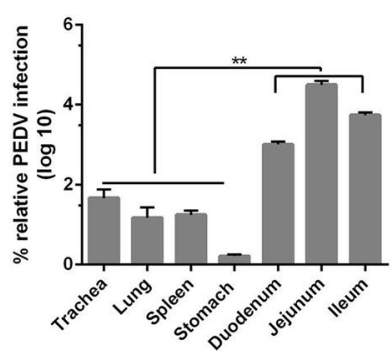

E

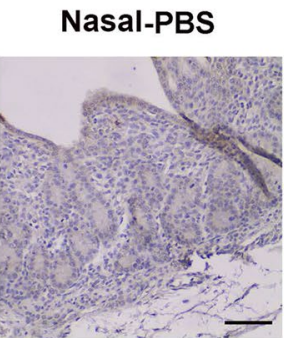

B

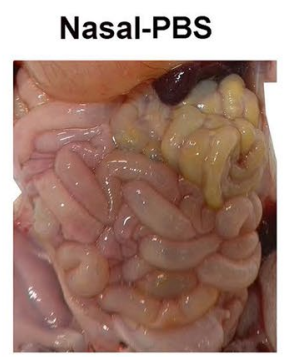

Nasal-PEDV

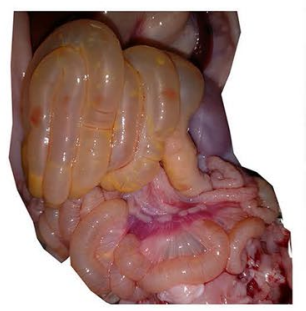

D

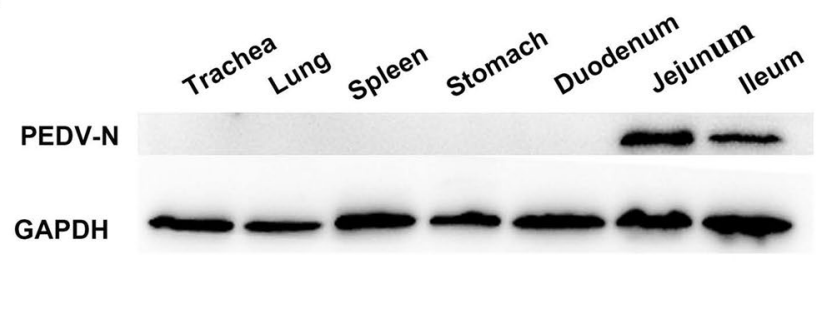

F

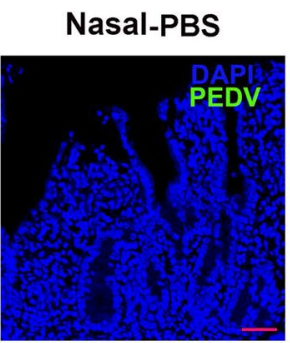

Nasal-PEDV

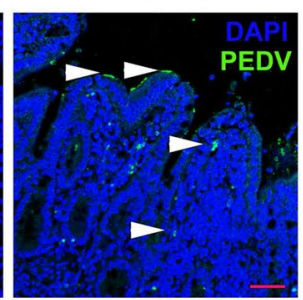

Figure7 The distribution of PEDV in neonatal piglets after intranasal inoculation. A Acute watery diarrhea in neonatal piglets after PEDV intranasal inoculation at 56hpi (white arrowhead). B Pathological changes in the intestine after the autopsy from different groups (Nasal-PBS, Nasal-PEDV). C RNA expression levels of PEDV in different tissues of piglets at $60 \mathrm{hpi}$ after PEDV intranasal inoculation. Data are the mean \pm SD. Statistical significance was tested using one-way ANOVA. ${ }^{*} P<0.01$. D Protein expression of PEDV in different tissues of diarrheic piglets a determined by western blotting with a mouse $m A b$ against $N$ protein. At least three independent experiments were performed. $\mathbf{E}, \mathbf{F} I H C$ and IFA of the intestine of intranasally inoculated piglets at $60 \mathrm{~h}$. PEDV antigen was located in the jejunum villus (white arrowhead). The scale bar represents $100 \mu \mathrm{m}$. Blue, DAPI; green, PEDV.

immune cells may sometimes be harnessed by viruses to help them overcome the epithelial barrier. When this happens, a pathway is created that allows viruses to enter the submucosal layer [26-28]. A pioneering study on cytomegalovirus infection via the intranasal route defined the nasal mucosa, a natural site of viral entry, as a novel site of viral persistence [29]. Measles virus infection in the macaque upper respiratory tract is mediated by subepithelial immune cells [30, 31]. PEDV can infect piglets through the nasal cavity, where DCs play an important role [12]. Presently, numerous $\mathrm{CD}^{+} \mathrm{T}$ cells were distributed in the subepithelium of the nasal cavity from neonatal piglets. This finding implies that PEDV in the nasal cavity may be exploited by $\mathrm{CD}^{+} \mathrm{T}$ cells beneath the nasal epithelium instead of DCs.
Most viruses normally replicate in the mucosal epithelial cells at the invasion site. However, this does not result in any cellular cytopathic changes. For example, porcine alpha-herpes virus pseudorabies virus can replicate in epithelial cells of the surface mucosa and spread to the whole body via the circulatory system. EpsteinBarr virus can replicate in nasopharyngeal epithelial cells and spread to the whole body via virus-carrying $B$ cells [32-35]. Although the replication of these viruses in mucosal epithelial cells does not produce cytopathic effects, they can serve as a source of infection with the potential to spread at any time [36, 37]. Similar to other studies, we found that PEDV could replicate in NECs and become a source of infection. PEDV-carrying 
NECs allow the virus to be transferred to $\mathrm{CD}^{+} \mathrm{T}$ cells beneath the NECs in neonatal piglets via cell-to-cell contact. The cell-to-cell transfer of PEDV could enable the virus to evade antibody neutralization.

Collectively, our results show that PEDV can cause typical PED symptoms in neonatal piglets through intranasal inoculation. PEDV can slowly replicate inside the nasal epithelium. This infection can induce an innate immune response in NECs, leading to changes in the expression of TLRs and cytokines. The viruses tend to be localized at the rear end of the nasal cavity. PEDV infected NECs allow the virus to be transferred to $\mathrm{CD}^{+} \mathrm{T}$ cells via cell-to-cell contact. Our results reveal the mechanism of intranasal inoculation of PEDV in neonatal piglets, which can provide more data on the development of effective strategies for controlling PEDV epidemics.

\section{Supplementary Information}

The online version contains supplementary material available at https://doi. org/10.1186/s13567-020-00883-w.

\section{Additional file 1. The identification of NECs.}

Additional file 2. Hematoxylin-eosin staining of the small intestine of the neonatal piglets.

\section{Abbreviations}

PEDV: porcine epidemic diarrhea virus; PED: porcine epidemic diarrhea; DCs: dendritic cells; PRRs: pattern recognition receptors; NECs: nasal epithelial cells; MOI: multiplicity of infection; FACS: fluorescence-activated cell sorting; TLR: toll-like receptor; CK18: cytokeratin 18; PVDF: polyvinylidene difluoride; hpi: hour postinfection; TGEV: transmissible gastroenteritis virus; PRRS: porcine Reproductive and Respiratory Syndrome; APC: anti-allophycocyanin; IHC: immunohistochemistry; IF: immunofluorescence; GAPDH: glyceraldehyde 3-phosphate dehydrogenase; RT-qPCR: quantitative reverse transcription-PCR; TCID: tissue culture infectious dose; IFN: interferon; IL: interleukin; TNF: tumor necrosis factor; NF-kB: nuclear factor-kappa B; SDS-PAGE: sodium dodecyl sulfate-polyacrylamide gel electrophoresis.

\section{Acknowledgements}

We thank Jiangsu Academy of Agricultural Sciences for providing us with the piglets.

\section{Authors' contributions}

Chen Yuan participated in performances of the experiments, analyzed the data and prepared the manuscript. Yuxin Jin, Yuchen Li and Penghao Zhang were responsible for isolating cells and a series of co-culture models establishment. En Zhang raised piglets and collected the samples. Qian Yang designed the study and revised the manuscript. All authors read and approved the final manuscript.

\section{Funding}

This work was supported by 31930109,31772777 and 32002261 from the National Natural Science Foundation of China and a Project Funded by the Priority Academic Program Development of Jiangsu Higher Education Institutions (PAPD).

\section{Availability of data and materials}

The datasets used and analyzed during the current study are available from the corresponding author on reasonable request.

\section{Ethics approval and consent to participate}

All efforts were made to minimize suffering. The animal protocol was approved by the University of Nanjing Agriculture University Committee on Animal Resources Committee (Permit Number: SYXK2011-0036).

\section{Consent for publication}

Not applicable.

\section{Competing interests}

The authors declare no competing financial interests.

Received: 1 September 2020 Accepted: 16 December 2020

Published online: 17 February 2021

\section{References}

1. Richard M, van den Brand JMA, Bestebroer TM, Lexmond P, de Meulder D, Fouchier RAM, Lowen AC, Herfst S (2020) Influenza A viruses are transmitted via the air from the nasal respiratory epithelium of ferrets. Nat Commun 11:766

2. Briles DE, Novak L, Hotomi M, van Ginkel FW, King J (2005) Nasal colonization with Streptococcus pneumoniae includes subpopulations of surface and invasive pneumococci. Infect Immun 73:6945-6951

3. Josefine P, Yuan Z, Olafsdottir TA, Karolina TR, Cairns TM, Frank W, Sattentau QJ, Eisenberg RJ, Cohen GH, Harandi AM (2016) Nasal immunization confers high avidity neutralizing antibody response and immunity to primary and recurrent genital herpes in guinea pigs. Front Immunol 7:640. https://doi.org/10.3389/fimmu.2016.00640

4. Bonifait L, Charlebois R, Vimont A, Turgeon N, Veillette M, Longtin Y, Jean J, Duchaine C (2015) Detection and quantification of airborne norovirus during outbreaks in healthcare facilities. Clin Infect Dis 61:299-304

5. Marks PJ, Vipond IB, Regan FM, Wedgwood K, Fey RE, Caul EO (2003) A school outbreak of Norwalk-like virus: evidence for airborne transmission. Epidemiol Infect 131:727-736

6. Vanders RL, Hsu A, Gibson PG, Murphy VE, Wark PAB (2019) Nasal epithelial cells to assess in vitro immune responses to respiratory virus infection in pregnant women with asthma. Respir Res 20:259

7. Cima G (2014) PED virus reinfecting U.S. herds Virus estimated to have killed 7 million-plus pigs. J Am Vet Med Assoc 245:166

8. Song D, Moon H, Kang B (2015) Porcine epidemic diarrhea: a review of current epidemiology and available vaccines. Clin Exp Vaccine Res 4:166-176

9. Song D, Park B (2012) Porcine epidemic diarrhoea virus: a comprehensive review of molecular epidemiology, diagnosis, and vaccines. Virus Genes 44:167

10. Sun RQ, Cai RJ, Chen YQ, Liang PS, Chen DK, Song CX (2012) Outbreak of porcine epidemic diarrhea in suckling piglets China. Emerg Infect Dis 18:161-163

11. Lin CM, Saif LJ, Marthaler D, Wang Q (2016) Evolution, antigenicity and pathogenicity of global porcine epidemic diarrhea virus strains. Virus Res 226:20-39

12. Li Y, Wu Q, Huang L, Yuan C, Wang J, Yang Q (2018) An alternative pathway of enteric PEDV dissemination from nasal cavity to intestinal mucosa in swine. Nat Commun 9:3811

13. Yang $Y$, Jing $Y$, Wang J, Yang Q (2018) Histological studies on the development of porcine tonsils after birth. J Morphol 279:1185-1193

14. Yang JJ, Dai L, Yu QH, Yang Q (2017) Histological and anatomical structure of the nasal cavity of Bama minipigs. PLoS One 12:e0173902. https://doi. org/10.1371/journal.pone.0173902

15. Emo KL, Hyun YM, Reilly E, Barilla C, Gerber S, Fowell D, Kim M, Topham DJ (2016) Live imaging of influenza infection of the trachea reveals dynamic regulation of $\mathrm{CD} 8(+) \mathrm{T}$ cell motility by antigen. PLoS Pathog 12:e1005881. https://doi.org/10.1371/journal.ppat.1005881

16. Zens KD, Connors T, Farber DL (2017) Tissue compartmentalization of T cell responses during early life. Semin Immunopathol 39:593-604

17. Krejci J, Nechvatalova K, Blahutkova M, Faldyna M (2013) The respiratory tract in pigs and its immune system: a review. Vet Med 58:206-220

18. Denney L, Ho LP (2018) The role of respiratory epithelium in host defence against influenza virus infection. Biomed J 41:218-233 
19. Kawaguchi M, Kokubu F, Kuga H, Tomita T, Matsukura S, Suzaki H, Huang SK, Adachi M (2001) Influenza virus A stimulates expression of eotaxin by nasal epithelial cells. Clin Exp Allergy 31:873-880

20. Roberts N, MubarakAl R, Francisco D, Kraft M, Chu HW (2018) Comparison of paired human nasal and bronchial airway epithelial cell responses to rhinovirus infection and IL-13 treatment. Clin Transl Med 7:e13. https:// doi.org/10.1186/s40169-018-0189-2

21. Yu L, Dong J, Wang Y, Zhang P, Liu Y, Zhang L, Liang P, Wang L, Song C (2019) Porcine epidemic diarrhea virus nsp4 induces pro-inflammatory cytokine and chemokine expression inhibiting viral replication in vitro. Arch Virol 164:1147-1157

22. Cao L, Ge X, Gao Y, Ren Y, Ren X, Li G (2015) Porcine epidemic diarrhea virus infection induces NF-kappaB activation through the TLR2, TLR3 and TLR9 pathways in porcine intestinal epithelial cells. J Gen Virol 96:1757-1767

23. Wang F, Wang SQ, Wang HF, Wu ZC, Bao WB, Wu SL (2020) Effects of porcine epidemic diarrhea virus infection on Toll-like receptor expression and cytokine levels in porcine intestinal epithelial cells. Pol J Vet Sci 23:119-126

24. Qin T, Yin Y, Huang L, Yu Q, Yang Q (2015) H9N2 influenza whole inactivated virus combined with polyethyleneimine strongly enhances mucosal and systemic immunity after intranasal immunization in mice. Clin Vaccine Immunol 22:421-429

25. Maroof A, Yorgensen YM, Li Y, Evans JT (2014) Intranasal vaccination promotes detrimental Th17-mediated immunity against influenza infection. PLoS Pathog 10:e1003875

26. Murooka TT, Deruaz M, Marangoni F, Vrbanac VD, Seung E, von Andrian UH, Tager AM, Luster AD, Mempel TR (2012) HIV-infected T cells are migratory vehicles for viral dissemination. Nature 490:283-287

27. Yavlovich A, Viard M, Zhou M, Veenstra TD, Wang JM, Gong W, Heldman E, Blumenthal R, Raviv Y (2012) Ectopic ATP synthase facilitates transfer of HIV-1 from antigen-presenting cells to CD4(+) target cells. Blood 120:1246-1253

28. Harman AN, Kim M, Nasr N, Sandgren KJ, Cameron PU (2013) Tissue dendritic cells as portals for HIV entry. Rev Med Virol 23:319-333

29. Zhang S, Caldeira-Dantas S, Smith CJ, Snyder CM (2019) Persistent viral replication and the development of T-cell responses after intranasal infection by MCMV. Med Microbiol Immunol 208:457-468
30. Ludlow M, Lemon K, de Vries RD, McQuaid S, Millar EL, van Amerongen G, Yuksel S, Verburgh RJ, Osterhaus AD, de Swart RL, Duprex WP (2013) Measles virus infection of epithelial cells in the macaque upper respiratory tract is mediated by subepithelial immune cells. J Virol 87:4033-4042

31. Ludlow M, de Vries RD, Lemon K, McQuaid S, Millar E, van Amerongen G, Yuksel S, Verburgh RJ, Osterhaus A, de Swart RL, Duprex WP (2013) Infection of lymphoid tissues in the macaque upper respiratory tract contributes to the emergence of transmissible measles virus. J Gen Virol 94:1933-1944

32. Lamote JAS, Glorieux S, Nauwynck HJ, Favoreel HW (2016) The US3 protein of pseudorabies virus drives viral passage across the basement membrane in porcine respiratory mucosa explants. J Virol 90:10945

33. Nauwynck H, Glorieux S, Favoreel H, Pensaert M (2007) Cell biological and molecular characteristics of pseudorabies virus infections in cell cultures and in pigs with emphasis on the respiratory tract. Vet Res 38:229

34. Rosalespérez S, Canovaldez AM, Floresbalcázar CH, Guedeaedo F, Linosilva LS, Lozanoborbalas A, Navarromartín A, Poitevinchacón A (2014) Expression of Epstein-Barr virus-encoded latent membrane protein (LMP1), p16 and p53 proteins in nonendemic nasopharyngeal carcinoma (NPC): a clinicopathological study. Arch Med Res 45:229

35. Wang HB, Zhang H, Zhang JP, Li Y, Zhao B, Feng GK, Du Y, Xiong D, Zhong Q, Liu WL (2015) Neuropilin 1 is an entry factor that promotes EBV infection of nasopharyngeal epithelial cells. Nat Commun 6:6240

36. Jia L, Tsang C, Yip Y, Law W, Zhang J, Tsao G (2015) Establishment and characterization of immortalized nasopharyngeal epithelial cells with stable infection of Epstein-Barr virus. Can Res 75:2731-2731

37. Sandekian V, Lim D, Prud'homme P, Lemay G (2013) Transient high level mammalian reovirus replication in a bat epithelial cell line occurs without cytopathic effect. Virus Res 173:327-335

\section{Publisher's Note}

Springer Nature remains neutral with regard to jurisdictional claims in published maps and institutional affiliations.

Ready to submit your research? Choose BMC and benefit from

- fast, convenient online submission

- thorough peer review by experienced researchers in your field

- rapid publication on acceptance

- support for research data, including large and complex data types

- gold Open Access which fosters wider collaboration and increased citations

- maximum visibility for your research: over $100 \mathrm{M}$ website views per year

At BMC, research is always in progress.

Learn more biomedcentral.com/submissions 